11

\title{
Влияние электрического и магнитного полей на межзонную люминесценцию в полупроводниковых квантовых проволоках
}

\author{
(C) Э.П. Синявский ${ }^{1}$, Н.С. Костюкевич ${ }^{2}$ \\ ${ }^{1}$ Институт прикладной фризики Академии наук Молдовы, \\ MD-2028 Кишинев, Молдова \\ ${ }^{2}$ Приднестровский государственный университет имени Т.Г. Шевченко, \\ MD-3300 Тирасполь, Молдова \\ e-mail:gravitonchik@gmail.com
}

Поступила в редакцию 18.03.2019 г.

В окончательной редакции 06.06.2019 г.

Принята к публикации 11.06.2019 г.

Теоретически исследуются межзонные оптические переходы в квантовых проволоках в модели параболического потенциала в электрическом и магнитном полях, направленных перпендикулярно оси нанопроволоки. Рассчитаны частотные зависимости интенсивности люминесценции света при учете взаимодействия носителей с шероховатой поверхностью и с длинноволновыми акустическими колебаниями. Получены графики частотной зависимости интенсивности люминесценции при различных значениях электрического и магнитного полей, зависимости полуширины люминесценции от радиуса проволоки, а также проведено сравнение с экспериментом.

Ключевые слова: межзонные оптические переходы, нанопроволока, люминесценция, поглощение, шероховатая поверхность.

DOI: $10.21883 /$ OS.2019.11.48523.119-19

\section{Введение}

В настоящее время большой интерес представляют исследования оптических свойств размерно-ограниченных квантовых систем (квантовые проволоки, нанотрубки). Именно при исследовании, например, процессов люминесценции в таких одномерных системах могут быть очень важными процессы рассеяния носителей на шероховатой поверхности, которые могут полностью определять оптические свойства $[1,2]$, явления переноса в таких квантовых системах. Межзонная люминесценция экспериментально наблюдалась в квантовых ямах (КЯ) InGaAs/AlGaAs [3], GaAs/AlGaAs [4,5], в квантовых проволоках (КП) InN [6], а исследования зависимости интенсивной фотолюминесценции от размеров нанопроволоки $\mathrm{CdZnS}$ проводились в [7]. Частотная зависимость коэффициента поглощения света и интенсивности люминесценции экспериментально изучались в $[4,5,8]$. В последние годы интенсивно исследовались оптические свойства полупроводных КП типа $\mathrm{ZnO}, \mathrm{GaN}, \mathrm{CdS}, \mathrm{InP}$, GaAs [6], в которых, как показывают эксперименты, очень активны процессы рассеяния носителей на шероховатой поверхности. Оптические свойства КП Ge исследовались в [7] в широкой области изменения радиуса нанопроволоки $(R=100,250,1100 \AA)$. Привлекательность и актуальность исследований оптических свойств КП связана с тем, что в одномерных квантовых системах на дне каждой размерно-квантованной зоны проводимости (валентной зоны) возникают особенности в плотности электронных состояний. Именно это обстоятельство приводит к специфическим по сравнению с двумерными системами особенностям частотных зависимостей люминесценции и коэффициента поглощения электромагнитной волны. Важно отметить, что заметное влияние размерного квантования на оптические свойства КП возможно при больших радиусах нанопроволоки $\left(R \leq 10^{3} \AA\right)[8]$.

\section{Постановка задачи. Общие соотношения}

В настоящей работе подробно исследуется интенсивность зона-зонной люминесценции в КП (в модели параболического потенциала) в поперечных электрическом и однородном магнитном полях. Заметим, что описание кинетических свойств КП в модели параболического потенциала является вполне обоснованным $[9,10]$ и в настоящее время часто применяемым [11]. При исследовании оптических характеристик в конденсированных средах используется метод балансного уравнения. Изменение энергии $U$ электромагнитного поля в единицу времени в нижайшем приближении по электрон-фотонному взаимодействию определяется соотношением [12]

$$
\frac{d U}{d t}=\hbar \Omega\left\{(1+N) W_{l}-W_{p}\right\}
$$

Здесь $\hbar \Omega$ - энергия фотона, $N$ - число фотонов в единице объема, т.е. $N \hbar \Omega$ определяет энергию электромагнитного поля в единице объема.

Согласно (1), увеличение энергии электромагнитного поля (первое слагаемое) естественно связано с про- 
цессами люминесценции, а уменьшение (второе слагаемое) определяется процессами поглощения света. Интенсивность люминесценции связана с фурье-образом корреляционной функции произведения операторов импульса [12]:

$$
\begin{gathered}
W_{l}=\frac{2 \pi e^{2}}{m_{0}^{2} \hbar \Omega V} \int_{-\infty}^{\infty} d t e^{-i \Omega t}\langle(\hat{P}(t) \xi)(\hat{P} \xi)\rangle, \\
\hat{P}(t)=e^{\frac{i t \hat{H}}{\hbar}} \hat{P} e^{-\frac{i t \hat{H}}{\hbar}} .
\end{gathered}
$$

Здесь $\hat{H}-$ гамильтониан исследуемой квантовой системы в отсутствие электромагнитного поля частоты $\Omega$ и поляризации $\xi, m_{0}$ - масса свободного электрона, $V$ - объем исследуемой квантовой системы. Так как в дальнейшем рассматриваем взаимодействие носителей с длинноволновыми акустическими колебаниями и учитываем рассеяния носителей на шероховатой поверхности наноструктуры, то $\langle\ldots\rangle$ в (2) описывает усреднение с равновесной матрицей плотности электрон-фононной системы [13] и усреднение по реализации случайного процесса [14].

В дальнейшем исследуем процессы спонтанного излучения слабой электромагнитной волны, связанные с квантовыми переходами электрона из зоны проводимости $(c)$ в валентную зону $(v)$. В этом случае выражение для интенсивности люминесценции (2) в представлении вторичного квантования определяется соотношением

$$
\begin{aligned}
W_{l}= & \frac{2 \pi e^{2}}{\hbar \Omega V}\left|\frac{\hat{P}_{c v} \xi}{m_{0}}\right|^{2} \sum_{\alpha \beta \alpha_{1} \beta_{1}}\langle\alpha \mid \beta\rangle\left\langle\beta_{1} \mid \alpha_{1}\right\rangle \\
& \times \int_{-\infty}^{\infty} d t e^{-i \Omega t}\left\langle a_{\alpha}^{c+}(t) a_{\beta}^{v}(t) a_{\beta_{1}}^{v+} a_{\alpha_{1}}^{c}\right\rangle .
\end{aligned}
$$

Здесь введены следующие обозначения: $a_{\alpha}^{i+}\left(a_{\alpha}^{i}\right)-$ операторы рождения (уничтожения) электронов в состоянии $\alpha$ в $i$-й зоне, $\hat{P}_{c v}-$ матричный элемент оператора импульса на блоховских волновых функциях электрона в зоне проводимости и в валентной зоне, $\langle\alpha \mid \beta\rangle-$ матричный элемент на сглаженных волновых функциях зоны проводимости $(\langle\alpha|)$ и валентной зоны $(|\beta\rangle)$,

$$
\hat{a}_{\alpha}^{i}(t)=e^{\frac{i t \hat{H}}{\hbar}} \hat{a}_{\alpha}^{i} e^{-\frac{i t \hat{H}}{\hbar}}
$$

Если $\hat{V}$ - оператор взаимодействия электрона с акустическими фононами и (или) с шероховатой поверхностью $\left(\hat{H}_{i}=\hat{H}_{i}^{0}+\hat{V}, \hat{H}_{i}^{0}\left|\alpha^{i}\right\rangle=E_{i}\left|\alpha^{i}\right\rangle\right)$, то, согласно [13],

$$
\begin{gathered}
a_{\alpha}^{+c}(t)=\Sigma_{\alpha_{2}} a_{\alpha_{2}}^{+c}\left\langle\alpha_{2}\left|e^{i \frac{t}{\hbar} \hat{H}_{C}}\right| \alpha\right\rangle, \\
a_{\beta}^{v}=\Sigma_{\beta_{2}}\left\langle\beta\left|e^{-i \frac{t}{\hbar} \hat{H}_{V}}\right| \beta_{2}\right\rangle a_{\beta_{2}}^{v} .
\end{gathered}
$$

С учетом (5) соотношение (3) можно записать в следующем виде:

$$
\begin{aligned}
W_{l}= & \frac{2 \pi e^{2}}{\hbar \Omega V}\left|\frac{\hat{P}_{c v} \xi}{m_{0}}\right|^{2} \sum_{\alpha \beta \alpha_{1} \beta_{1}}\langle\alpha \mid \beta\rangle\left\langle\beta_{1} \mid \alpha_{1}\right\rangle \int_{-\infty}^{\infty} d t e^{-i \Omega t} n_{\alpha_{1}}^{(c)} \\
& \times\left(1-n_{\beta_{1}}^{(v)}\right)\left\{\left\langle\alpha_{1}\left|e^{\frac{i t \hat{H}_{c}}{\hbar}}\right| \alpha\right\rangle\left\langle\beta\left|e^{-\frac{i t \hat{H}_{v}}{\hbar}}\right| \beta_{1}\right\rangle\right\} .
\end{aligned}
$$

$n_{\alpha}^{c}\left(n_{\beta}^{v}\right)$ - равновесные функции распределения электронов (дырок) в размерно-квантованной зоне проводимости (в валентной зоне) исследуемой наносистемы, $\{\ldots\}$ описывает усреднение по системе свободного фононного поля и усреднение по реализации случайного процесса.

Усреднение по системе свободного фононного поля и по реализации случайного процесса проведем с использованием метода кумулянт и в приближении времени релаксации $[13,14]$ :

$$
\begin{gathered}
\left\{\left\langle\alpha_{1}\left|e^{\frac{i \hat{H}^{c}}{\hbar}} \alpha\right| \alpha\right\rangle\right\}=\delta_{\alpha_{1} \alpha} e^{\frac{i t \varepsilon_{\alpha}^{c}}{\hbar}} e^{-\Gamma_{\alpha}^{c}|t|}, \\
\left\{\left\langle\beta\left|e^{\frac{i t \hat{H}^{v}}{\hbar}} \beta_{1}\right| \alpha\right\rangle\right\}=\delta_{\beta \beta_{1}} e^{-\frac{i t \varepsilon_{\beta}^{v}}{\hbar}} e^{-\Gamma_{\alpha}^{v}|t|},
\end{gathered}
$$

$\varepsilon_{\alpha}^{c}\left(\varepsilon_{\beta}^{v}\right)$ - энергия электрона в зоне проводимости (в валентной зоне) в состоянии $\alpha ; 2 \Gamma_{\alpha}^{c}\left(2 \Gamma_{\beta}^{v}\right)$ определяет квантово-механическую вероятность рассеяния носителей в единицу времени в $c$-зоне (в $v$-зоне) на длинноволновых акустических колебаниях [13] и на шероховатой поверхности [14]. В рассматриваемых выше приближениях интенсивность межзонной спонтанной люминесценции (6) принимает окончательное выражение:

$$
\begin{aligned}
W_{l}= & \frac{2 \pi e^{2}}{\hbar \Omega V}\left|\frac{\hat{P}_{c v} \xi}{m_{0}}\right|^{2} \sum_{\alpha \beta}|\langle\alpha \mid \beta\rangle|^{2} n_{\alpha}^{c}\left(1-n_{\beta}^{v}\right) \\
& \times \frac{2\left(\Gamma_{\alpha}^{c}+\Gamma_{\beta}^{v}\right.}{\left(\Gamma_{\alpha}^{c}+\Gamma_{\beta}^{v}\right)^{2}+\frac{1}{\hbar^{2}}\left(\varepsilon_{\alpha}^{c}-\varepsilon_{\beta}^{v}-\hbar \Omega\right)^{2}} .
\end{aligned}
$$

\section{Обсуждение результатов}

В качестве примера исследуем процессы спонтанной зона-зонной люминесценции в полупроводниковых КП (в модели параболического потенциала) в присутствии поперечных электрическом Е и однородном магнитном Н полях. В рассматриваемом случае гамильтониан для электрона в зоне проводимости с эффективной массой $m_{e}$ в калибровке Ландау $\mathbf{A}(-H y, 0,0)$ записывается в виде $(\mathbf{H}\|O z, \mathbf{H}\| \mathbf{E})$

$$
\begin{aligned}
\hat{H}_{e}^{0}= & \frac{1}{2 m_{e}}\left\{\left[\hat{p}_{x}-\frac{e H y}{c}\right]^{2}+\hat{p}_{y}^{2}+\hat{p}_{z}^{2}\right\} \\
& +\frac{m_{e} \omega_{e}^{2}\left(y^{2}+z^{2}\right)}{2}+e E z .
\end{aligned}
$$

Здесь $\hbar \omega_{e}$ - энергия размерного квантования, которая простым образом связана с потенциальной энергией $\Delta E_{c}$ на границе КП радиуса $R, \omega_{e}=\frac{1}{R}\left[\frac{2 \Delta E_{c}}{m_{e}}\right]^{1 / 2}$. 
Волновые функции и собственные значения энергии вычисляются аналогично [10] и определяются соотношениями

$$
\begin{gathered}
\Psi_{\alpha}(x, y, z)=\frac{e^{i k_{x} x}}{\sqrt{L_{x}}}\left(\frac{\lambda_{1} \lambda_{2}{ }^{1 / 4}}{\pi^{2}} \frac{1}{\sqrt{2^{n} n ! 2^{m} m !}} H_{m}\right. \\
\times\left[\left(z+z_{0}\right) \sqrt{\lambda_{1}}\right] e^{-\frac{\lambda_{1}}{2}\left(z+z_{0}\right)^{2}} H_{n}\left[\left(y-y_{0}\right) \sqrt{\lambda_{2}}\right] e^{-\frac{\lambda_{2}}{2}\left(y-y_{0}\right)^{2}}, \\
E_{\alpha}^{c}=\frac{\hbar^{2} k_{x}^{2}}{2 m_{e}^{*}}+\hbar \Omega_{e}\left(n+\frac{1}{2}\right)+\hbar \omega_{e}\left(m+\frac{1}{2}\right)-\Delta_{c}, \\
\lambda_{1}=\frac{m_{e} \omega_{e}}{\hbar}, \quad \lambda_{2}=\frac{m_{e} \Omega_{e}}{\hbar}, \\
\Omega_{e}=\left(\omega_{e}^{2}+\omega_{c}^{2}\right)^{1 / 2}, \quad m_{e}^{*}=m_{e}\left(\frac{\Omega_{e}}{\omega_{e}}\right)^{2},
\end{gathered}
$$

$\Delta_{c}=\frac{e^{2} E^{2}}{2 m_{e} \omega_{e}^{2}}, \quad z_{0}=\frac{e E}{m_{e} \omega_{e}^{2}}, \quad y_{0}=\frac{k_{x} \hbar \omega_{c}}{m_{e} \Omega_{e}^{2}}, \quad \omega_{c}=\frac{e H}{m_{e} c}$,

$H_{n}(z)$ - полиномы Эрмита-Чебышева, $L_{x}$ - длина КП, $k_{x}$ - волновой вектор частицы вдоль оси нанопроволоки.

Аналогично можно вычислить собственные значения для носителей с эффективной массой $m_{v}$ в валентной зоне:

$$
\begin{gathered}
E_{\beta}^{v}=-\frac{\hbar^{2} k_{x}^{2}}{2 m_{v}^{*}}-\hbar \Omega_{v}\left(n+\frac{1}{2}\right)-\hbar \omega_{v}\left(m+\frac{1}{2}\right)+\Delta_{v}-E_{g} \\
\Omega_{v}=\left(\omega_{v}^{2}+\omega_{c} v^{2}\right)^{1 / 2}, \quad \Delta_{v}=\frac{e^{2} E^{2}}{2 m_{v} \omega_{v}^{2}} \\
m_{v}^{*}=m_{v}\left(\frac{\Omega_{v}}{\omega_{v}}\right)^{2}, \quad \omega_{c} v=\frac{e H}{m_{v} c}
\end{gathered}
$$

$\hbar \omega_{v}$ - энергия размерного квантования в валентной зоне, $E_{g}$ - ширина запрещенной зоны объемного материала. Волновые функции для носителей в валентной зоне записываются аналогично (10), но $m_{e}$ заменяется на $m_{v}, \omega_{e}-$ на $\omega_{v}, \Omega_{e}-$ на $\Omega_{v}, E-$ на $-E$.

В дальнейшем рассмотрим взаимодействие носителей с шероховатой поверхностью и учитываем взаимодействие электронов с длинноволновыми акустическими колебаниями. Квантово-механическая вероятность рассеяния носителей в единицу времени в приближении времени релаксации $[13,14]$ определяется следующим образом:

$$
\begin{gathered}
\Gamma_{\alpha}^{c}=\frac{1}{\left|k_{x}\right|} \gamma, \\
\gamma=\frac{E_{1}^{2} k_{0} T m_{e}^{2} \omega_{e}}{2 \pi \hbar^{4} \rho v^{2}}\left(1+\delta^{2}\right)^{5 / 4}+\frac{2 m_{e} \gamma_{0} \omega_{e}^{2}}{\hbar R^{2}}\left(1+\delta^{2}\right) \\
\times\left[\frac{1}{2}\left(\frac{1}{\sqrt{1+\delta^{2}}}\right)+\frac{2 \Delta_{C}}{\hbar \omega_{e}}\right]^{2}, \\
\delta=\frac{\hbar \omega_{c}}{\hbar \omega_{e}} .
\end{gathered}
$$

Здесь введены следующие обозначения: $E_{1}-$ константа деформационного потенциала для электрона, $v-$ скорость звука в наноструктуре с плотностью $\rho, \gamma_{0}^{1 / 3}$ определяет высоту флуктуаций.

Как непосредственно следует из (13), время релаксации, определяемое взаимодействием электрона с шероховатой поверхностью (второе слагаемое в (13)), существенно зависит от радиуса КП $\left(\propto 1 / R^{4}\right)$, и, следовательно, его необходимо учитывать при исследовании кинетических явлений в нанопроволоках с малыми $R$. Для типичных полупроводниковых КП типа GaAs $\left(m_{e}=0.06 m_{0}, m_{v}=0.4 m_{0}, E_{1}=10 \mathrm{eV}\right.$, $\left.\left.\Delta E_{c}=0.255 \mathrm{eV}, \quad \rho=5.4 \mathrm{~g} / \mathrm{cm}^{3}\right), \quad v=3 \cdot 10^{5} \mathrm{~cm} / \mathrm{s}\right) \quad$ при $\gamma_{0}^{1 / 3}=20 \AA$ (при таких значениях $\gamma_{0}$ подвижность достигает больших экспериментально наблюдаемых значений: $\left.\mu \sim 3 \cdot 10^{4} \mathrm{~cm}^{2} /(\mathrm{V} \cdot \mathrm{s})\right)$ рассеянием электронов на шероховатой поверхности можно пренебречь, если $R>5 \cdot 10^{2} \AA$. В этом случае время релаксации определяется рассеянием носителей на длинноволновых акустических колебаниях (первое слагаемое в (13) $\gamma \sim 1 / R$, т. е. значительно слабее зависит от размеров наноструктуры. Из явного вида $\gamma$ следует, что с ростом напряженности магнитного поля $\gamma$ увеличивается, что связано с увеличением локализации носителей. В поперечном электрическом поле электроны „прижимаются“ к поверхности КП, что и приводит к заметному усилению процессов рассеяния частиц на шероховатой поверхности (рост второго слагаемого в (13) при увеличении Е).

Если подставить (12) в (8), то в случае невырожденного электронного (дырочного) газа интенсивность люминесценции, связанная с переходом электрона из нижайшего размерно-квантованного состояния зоны проводимости в нулевое размерно-квантованное состояние валентной зоны, принимает следующий вид:

$$
\begin{aligned}
& W_{l}=W_{0} I(\Delta), \\
& W_{0}=\frac{2 \pi e^{2}}{\Omega S}\left|\frac{\left.\hat{P}_{c v} \xi\right)}{m_{0}}\right|^{2} \Sigma_{\alpha \beta}\left|\left\langle 0_{c} \mid 0_{v}\right\rangle\right|^{2} \\
& \times \frac{n_{e} n_{v}}{k_{0} T}\left(\frac{2 \hbar}{\omega_{f}\left(m_{e}^{*}+m_{v}^{*}\right)}\right)^{1 / 2}, \\
& I(\Delta)=\int_{-\infty}^{\infty} d x e^{-\beta \hbar \omega_{f} x} \frac{1}{1+x\left[\frac{\Delta}{\hbar \omega_{f}}+x\right]^{2}}, \\
& \omega_{f}=\left[\frac{\hbar \gamma^{2}}{2 \mu^{*}}\right]^{1 / 3}, \quad \frac{1}{\mu^{*}}=\frac{1}{m_{e}^{*}}+\frac{1}{m_{v}^{*}}, \\
& \Delta=-\hbar \Omega+E_{g}^{*}, \quad \beta=\frac{1}{k_{0} T}, \\
& \Delta_{c v}=\frac{(e E R)^{2}}{4}\left(\frac{1}{\Delta E_{c}}+\frac{1}{\Delta E_{v}}\right), \\
& \left|\left\langle 0_{c} \mid 0_{v}\right\rangle\right|^{2}=4\left[\frac{R_{e} R_{v} R_{e}^{0} R_{v}^{0}}{\left(R_{e}^{2}+R_{v}^{2}\right)\left(R_{e}^{02}+R_{v}^{02}\right)}\right] \\
& \times \exp \left\{-\frac{e^{2} E^{2} R^{4}}{\left(R_{e}^{02}+R_{v}^{02}\right)}\left(\frac{1}{\Delta E_{c}}+\frac{1}{\Delta E_{v}}\right)^{2}\right\} \text {, }
\end{aligned}
$$




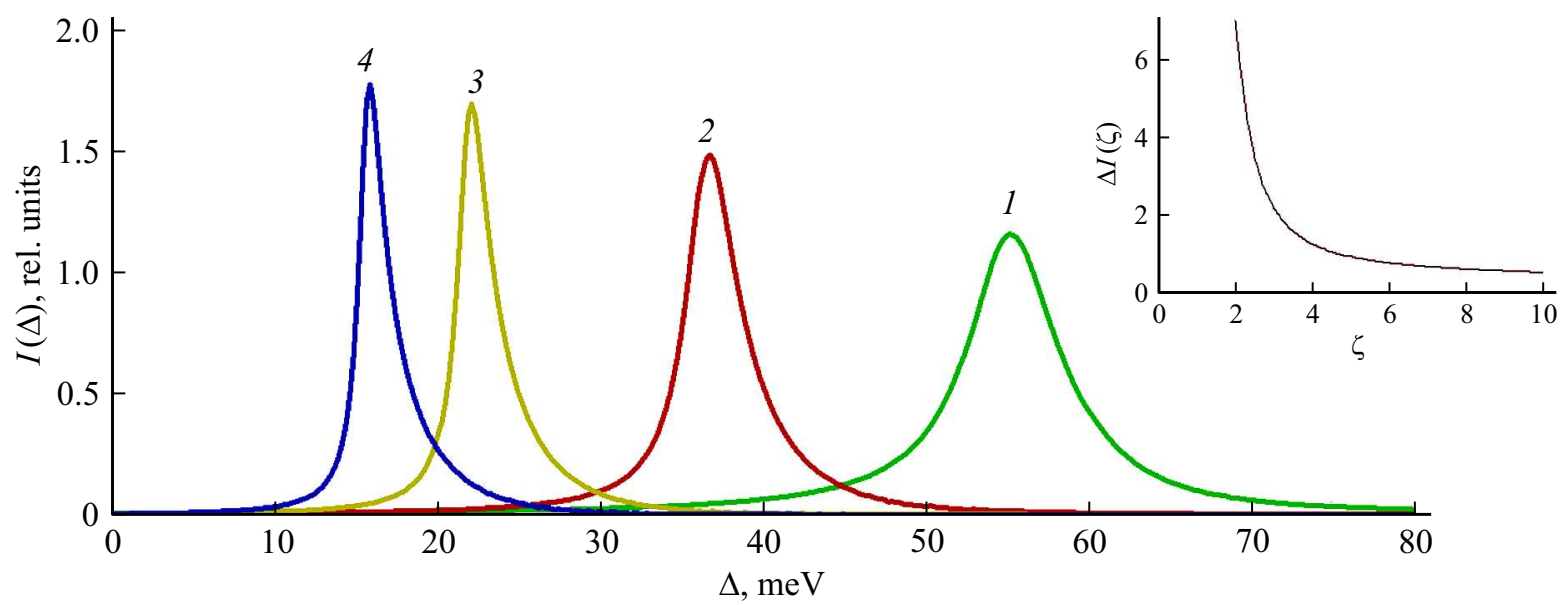

Рис. 1. Частотная зависимость интенсивности люминесценции (в относительных единицах) при $E=0, H=0$. Кривые $1-4$ получены соответственно при $\xi=2,3,5,7$. Во вставке представлена зависимость полуширины линии излучения от радиуса КП.

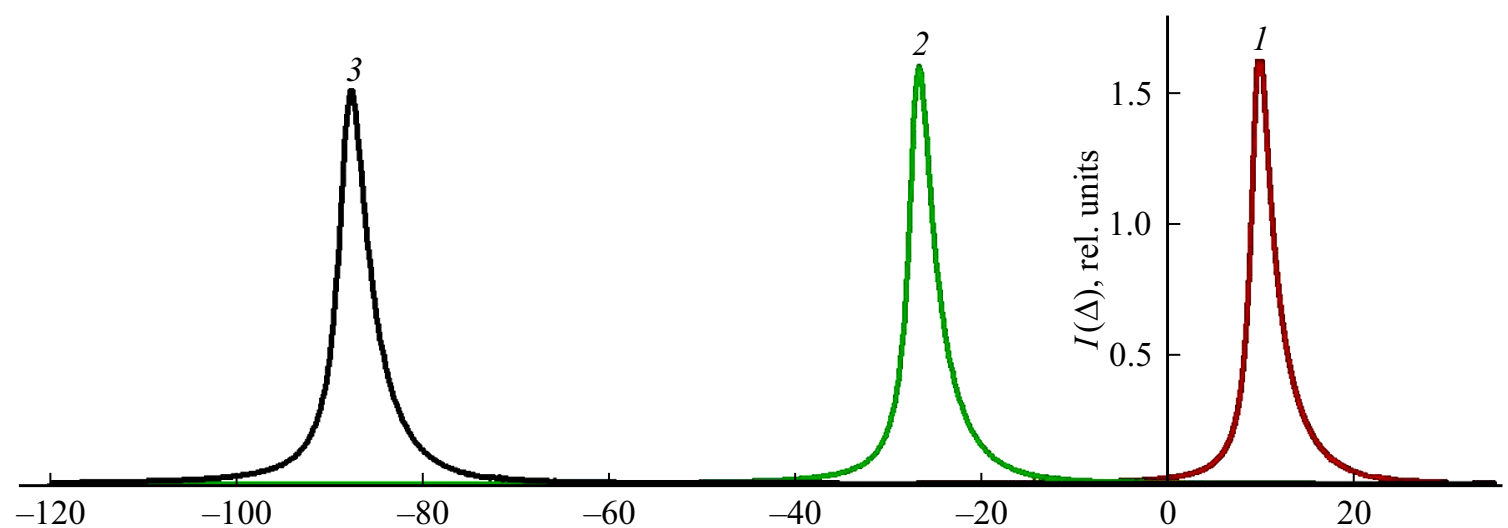

$\Delta, \mathrm{meV}$

Рис. 2. Частотная зависимость интенсивности люминесценции (в относительных единицах) при $H=0$, $\xi=5$. Кривые $1-3$ вычислены соответсвенно при $E=2 \cdot 10^{4}, 4 \cdot 10^{4}, 6 \cdot 10^{4} \mathrm{~V} / \mathrm{cm}$.

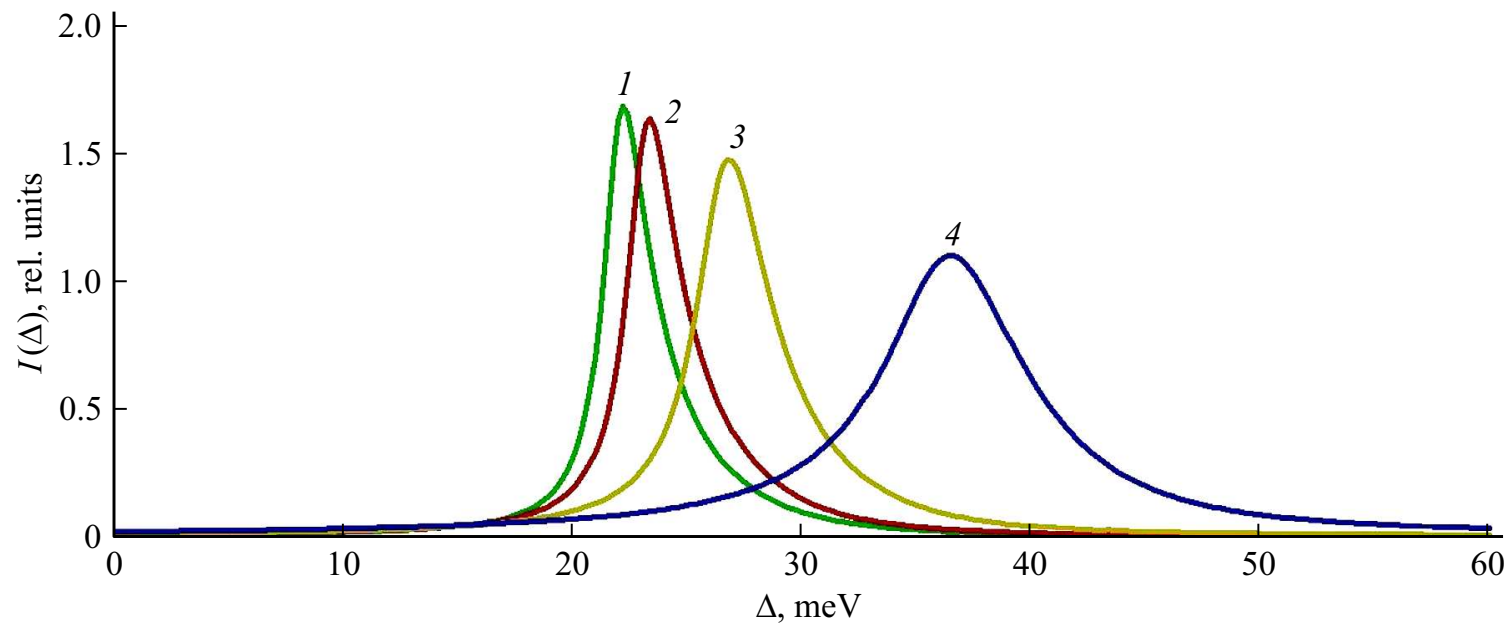

Рис. 3. Частотная зависимость интенсивности люминесценции (в относительных единицах) в присутствии магнитного поля (при $E=0, \xi=5)$. Кривые $1-4$ получены при $\delta=0.2,0.5,1,2$ соответственно. 


$$
\begin{aligned}
& R_{e}^{2}=\frac{\hbar}{\left(m_{e} \Omega_{e}\right.}, \quad R_{e}^{02}=\frac{\hbar}{m_{e} \omega_{e}} \\
& R_{v}^{2}=\frac{\hbar}{m_{v} \Omega_{v}}, \quad R_{v}^{02}=\frac{\hbar}{m_{v} \omega_{v}}
\end{aligned}
$$

$n_{e}\left(n_{v}\right)=\frac{N_{e}}{L_{x}}\left(\frac{N_{v}}{L_{x}}\right)$ - линейная концентрация электронов (дырок) в КП, $E_{g}^{*}=E_{g}+\frac{1}{2}\left(\hbar \omega_{e}+\hbar \Omega_{e}+\hbar \omega_{v}+\right.$ $\left.+\hbar \Omega_{v}\right)-\Delta_{c} v-$ ширина запрещенной зоны исследуемой наноструктуры во внешних полях, $S$ - сечение КП.

На рис. 1 представлена частотная зависимость интенсивности люминесценции (в относительных единицах) $(H=0, E=0, T=40 \mathrm{~K})$. Кривые $1-4$ получены при $\xi=2,3,5,7$ соответственно $\left(R=10^{2} \xi \AA\right)$. Как непосредственно следует из рис. 1 , полуширина линии спонтанной люминесценции с ростом радиуса нанопроволоки уменышается. На вставке рис. 1 приведена зависимость полуширины линии интенсивности люминесценции $\Delta I(\Delta)$ от радиуса КП. Следовательно, при малых радиусах КП $(\xi<4)$ полуширина резко уменьшается с ростом $R$. Именно в таких наносистемах рассеяние носителей на шероховатой поверхности является доминирующим механизмом рассеяния и $\omega_{f} \sim R^{-\frac{8}{3}}$. При $R=200,500$ и $700 \AA$ полуширина линии излучения $\Delta I$ соответственно равна $\Delta I=10.17,2.4$ и $1.9 \mathrm{meV}$. C ростом $R$ влияние рассеяния носителей на шероховатой поверхности на интенсивность люминесценции уменьшается, и полуширина линии излучения определяется взаимодействием заряженных частиц с длинноволновыми акустическими колебаниями $\left(\Delta I \propto R^{-2 / 3}\right)$ и, следовательно, слабее зависит от размеров исследуемой наноструктуры. Именно такую динамику изменения полуширины линии зона-зонной люминесценции от $R$ экспериментально наблюдали при исследовании магнитооптики КП [15]. С уменьшением радиуса КП ширина запрещенной зоны наноструктуры увеличивается на величину $\left(\hbar \omega_{e}+\hbar \omega_{v}\right)$, что, естественно, приводит к тому, что максимум зона-зонной люминесценции с уменьшением $R$ сдвигается в высокочастотную область спектра.

При уменьшении радиуса нанопроволоки увеличивается энергия размерного квантования в зонах $\left(\hbar \omega_{e}\right.$, $\left.\hbar \omega_{v} \propto \frac{1}{R}\right)$, что приводит к уменьшению числа размерноквантованных уровней в параболической потенциальной яме высоты $\Delta E_{c}\left(\Delta E_{v}\right)$. Последнее обстоятельство может оказаться причиной уменьшения числа пиков межзонного поглощения слабой электромагнитной волны. Именно такая динамика изменения спектра межзонного поглощения света наблюдалась в нанопроволоках $\mathrm{Ge}$ при изменении радиуса квантовой системы от $10^{2}$ до $1.1 \cdot 10^{3} \AA$ [7]. На рис. 2 приведена частотная зависимость интенсивности люминесценции (в относительных единицах, $H=0, T=40 \mathrm{~K}$ ) при различных значениях напряженности поперечного электрического поля $E$. Кривые $1-3$ вычислены при $E=2 \cdot 10^{3}, 4 \cdot 10^{3}$ и $6 \cdot 10^{3} \mathrm{~V} / \mathrm{cm}$ соответственно. Как непосредственно следует из рис. 2, с ростом $\mathbf{E}$ интенсивность спонтанной люминесценции уменьшается. Это связано с тем, что при увеличении $\mathbf{E}$ перекрывание сглаженных волновых функций зоны проводимости и валентной зоны уменьшается. Именно такое поведение интенсивности люминесценции при изменении напряженности поперечного поля наблюдалось в КП $\mathrm{CdS}, \mathrm{GaN}$ [16]. Если Е увеличивается, то ширина запрещенной зоны уменьшается на величину $\Delta_{c v}$. Это обстоятельство приводит к тому, что с ростом $\mathbf{E}$ максимум зона-зонной люминесценции сдвигается в длинноволновую область спектра (рис. 2).

На рис. 3 представлена частотная зависимость интенсивности зона-зонной спонтанной люминесценции (в относительных единицах) при различных значениях напряженности однородного магнитного поля. Кривые 1 4 получены при $\delta=0.2,0.5,1,2$ соответственно. При увеличении напряженности однородного магнитного поля ширина запрещенной зоны исследуемой наноструктуры увеличивается на $\frac{1}{2}\left(\hbar \Omega_{e}+\hbar \Omega_{v}\right)$, что естественно приводит к смещению максимума люминесценции в коротковолновую область спектра (рис. 3). С ростом Н взаимодействие электронов с шероховатой поверхностью и с длинноволновыми акустическими колебаниями увеличивается, как следует из (13), что и приводит к росту полуширины линии зона-зонной люминесценции.

\section{Заключение}

Проведены исследования по влиянию внешних полей (поперечных однородного магнитного и постоянного электрических полей) на интенсивность межзонной люминесценции в КП. Показано, что учет взаимодействия электрона с акустическими колебаниями и с шероховатой поверхностью наносистем позволяет описать зависимость полуширины линии люминесценции от радиуса КП, экспериментально наблюдаемую в одномерных квантовых системах.

\section{Благодарность}

Авторы приносят благодарность STCU (грант 6219) за частичную финансовую поддержку работы.

\section{Конфликт интересов}

Авторы заявляют, что у них нет конфликта интересов.

\section{Список литературы}

[1] Синявский Э.П., Костюкевич Н.С. // Опт. и спектр. 2013. T. 114. № 2. C. 205.

[2] Unuma T., Takahashi T., Noda T., Yoshita M., Sakaki H., Baba M., Akiyama H. // Appl. Phys. Lett. 2001. V. 78. N 22. P. 3448

[3] Brunner K., Bockelmann U., Abstreiter G., Walther M., Böhm G., Tränkle G., Weimann G. // J. Phys. IV. 1993. V. 03. N C5. P. 107. 
[4] Aiswarya Raj A.S., Biju V. // Mater. Sci. Semicond. Process. 2017. V. 68. C. 38.

[5] Pathak T.K., Swart H.C., Kroon R.E. // Spectrochim. Acta. A. Mol. Biomol. Spectrosc. 2018. V. 190. N 5. P. 164.

[6] Warburton R.J., Weilhammer K., Kotthaus J.P., Thomas M., Kroemer H. // Phys. Rev. Lett. 1998. V. 80. N 10. P. 2185.

[7] Cao L., White J.S., Park J., Schuller J.A., Clemens B.M., Brongersma M.L. // Nat. Mater. 2009. V. 8. N 8. P. 643.

[8] Agarwal R., Lieber C.M. // Appl. Phys. A. 2006. V. 85. N 3. P. 209.

[9] Beenakker C.W.J., Houten H., Ehrenreich H., Turnbull D. Solid State Physics: Semiconductor Heterostructures and Nanostructures. Academic Press, 1991. V. 44. 228 p.

[10] Гейлер В.А., Маргулис В.А., Филина Л.И. // ЖЭТФ. 1998. T. 86. № 4. C. 751.

[11] Гейлер В.А., Маргулис В.А. // ФТП. 1999. Т. 9. С. 1141.

[12] Белоусов А.В., Коварский В.А., Синявский Э.П. Оптические свойства молекулярных систем в поле низкочастотного лазерного излучения. Кишинев: Штиинца, 1989. $128 \mathrm{c}$.

[13] Синявский Э.П., Хамидуллин Р.А. // ФТП. 2002. Т. 36. № 8. C. 989.

[14] Синявский Э.П., Карапетян С.А. // ФТП. 2012. Т. 46. № 8. C. 1032.

[15] Кулаковский В.Д., Бутов Л.В. // УФН. 1995. Т. 165. № 2. C. 229.

[16] Greytak A.B., Barrelet C.J., Li Y., Lieber C.M. // Appl. Phys. Lett. 2005. T. 87. N 15. P. 151103. 